\title{
Perfluorooctane sulfonate induces heart toxicity involving cardiac apoptosis and inflammation in rats
}

\author{
DONGMIN XU ${ }^{1}, \mathrm{LI} \mathrm{LI}^{1}$, LEILEI TANG ${ }^{2}$, MING GUO $^{3 *}$ and JIE YANG ${ }^{3 *}$ \\ ${ }^{1}$ Department of Pharmacy, Chun'an First People's Hospital, Zhejiang Province People's Hospital \\ Chun'an Branch, Hangzhou, Zhejiang 311700; Departments of ${ }^{2}$ Pharmacy and ${ }^{3}$ Cardiology, \\ Zhejiang Xiaoshan Hospital, Hangzhou, Zhejiang 311200, P.R. China
}

Received October 15, 2020; Accepted June 11, 2021

DOI: $10.3892 /$ etm.2021.10936

\begin{abstract}
Perfluorooctane sulfonate (PFOS) is a persistent pollutant that exerts toxicity and induces cardiogenesis in humans and animals. Yet, the effect of PFOS exposure on cardiac toxicity in adult rats has, to our knowledge, not been reported and the mechanism still remains unknown. The present study aimed to investigate the toxicity of PFOS on rat hearts and any associated mechanisms. Rats were exposed to 0 (control), 1 and $10 \mathrm{mg} / \mathrm{kg}$ PFOS every other day for 14 days. Body weight and heart weight were recorded. The serum levels of lactic dehydrogenase (LDH), creatine kinase (CK), creatine kinase-isoenzyme-MB (CK-MB) and cardiac troponin-T (cTn-T) in heart tissues were measured using biochemical assays. TUNEL staining and western blotting were applied to analyze levels of apoptosis in rat hearts. Pathological assessment and immunohistochemistry analysis of heart tissues were used to evaluate the levels of PFOS-induced cardiotoxicity and inflammatory infiltration. PFOS exposure at the dosage of $10 \mathrm{mg} / \mathrm{kg}$ significantly increased the percentage of heart to body weight; however, it did not alter the body weight. At $10 \mathrm{mg} / \mathrm{kg}$, PFOS significantly increased expression levels of myocardial injury markers, such as cTn-T, LDH, CK and CK-MB, while $1 \mathrm{mg} / \mathrm{kg}$ PFOS upregulated the expression level of cTn-T in rats. Notably, cardiac fibrosis and myocardiac hypertrophy appeared in the $10 \mathrm{mg} / \mathrm{kg}$ PFOS group. In addition, TUNEL-positive cells were significantly increased by exposure to $10 \mathrm{mg} / \mathrm{kg}$ PFOS in rat heart tissues. The protein expressions profiles of p53 and Bax were also significantly upregulated in the $10 \mathrm{mg} / \mathrm{kg}$ PFOS group. Inflammatory infiltration, detected by anaylzing expression levels of IL-1 $\beta$
\end{abstract}

Correspondence to: Professor Jie Yang, Department of Cardiology, Zhejiang Xiaoshan Hospital, 728 Yucai North Road, Hangzhou, Zhejiang 311200, P.R. China

E-mail: 18967168829@163.com

*Joint senior authorship

Key words: perfluorooctane sulfonate, cardiac toxicity, myocardial injury, apoptosis, inflammation and TNF- $\alpha$, was significantly raised by $10 \mathrm{mg} / \mathrm{kg}$ PFOS exposure. In conclusion, these results demonstrated that $10 \mathrm{mg} / \mathrm{kg}$ PFOS-induced cardiac toxicity in rats, which was associated with an increase in apoptosis and the expression of proinflammatory cytokines.

\section{Introduction}

Perfluorooctane sulfonate (PFOS) is a degradation product of perfluorinated compounds and is characterized by widespread use and environmental stability (1). This chemical compound has been used in a large variety of industrial and commercial material, such as plastic packaging bags, cosmetics and textiles (2). It is globally distributed and can be detected in soil, air, water, wildlife and in humans $(1,2)$. It can accumulate biologically through the food chain, having a half-life of $\sim 5$ years in human serum (3). Therefore, the potential toxicity of PFOS is concerning. The chief manufacturer of PFOS in the United States, The 3M Company, is to halt production (4), and a series of international regulations were set out to restrict usage of polyfluoroalkyl substances in 2016 (5). However, emission of PFOS still persists in Asian markets (6,7). A recent study reported that PFOS concentrations in serum are significantly increased with age in the general Chinese population, suggesting that they may have common exposure sources (8).

As previously reported, PFOS involves several toxic effects in the cardiovascular (9) and reproductive systems (10), effecting immunological (11) and hepatic functions (12). Notably, the heart exhibits the second greatest bioaccumulation of PFOS after the liver, and it is also a target organ for PFOS. Our previous study indicated that several marker proteins involved in cardiovascular development, such as Brachyury, GATA4, myocyte enhancer factor $2 \mathrm{C}$ and $\alpha$-actinin, were downregulated when exposed to PFOS (13). In a marine medaka model and in embryonic stem cell (ESC)-derived cardiomyocytes, prenatal PFOS exposure disrupts the expression of genes associated with cardiac development, and affects the function of the heart $(9,14)$. PFOS has been considered to induce cardiac mitochondrial damage and gene transcript disorder (15), which may be one possible toxicity mechanism. Nevertheless, to our knowledge, the possible impact of PFOS on cardiac dysfunction in adult rats has not been investigated, and the underlying toxicity mechanism has not yet been fully elucidated. 
Apoptosis is a type of programmed cell death that participates in various pathological events (16). It has been widely reported that excessive apoptosis is responsible for structural abnormality and dysfunction of the heart $(17,18)$. During all stages of heart development, PFOS possesses the potential to alter key genes, reduce ATP production, stimulate reactive oxygen species (ROS) generation and induce apoptosis (19). In a zebrafish embryo model, PFOS exposure induced apoptosis and upregulated gene expression levels of P53 and Bax, which are associated with apoptosis. These genes are also closely associated with the JNK and p38 signaling pathway $(20,21)$. In addition, a potential association between PFOS and inflammation has been revealed in a previous study (22); however, the specific mechanism is unclear. PFOS can modulate the inflammatory factors, such as TNF- $\alpha$ and IL-6, in vivo and in vitro (22). Therefore, it was hypothesized that PFOS toxicity in the cardiac tissue of adult rats might be associated with inflammation and apoptosis.

The current study aimed to explore whether PFOS exposure would induce heart impairment and a degree of pathological change in rats. Moreover, the level of apoptosis and inflammatory infiltration in cardiomyocytes was investigated to provide evidence for further research on PFOS-induced cardiac toxicology.

\section{Materials and methods}

Ethics statement. Animal experiments in the current study were performed in accordance with the National Institutes of Health Guide for the Care and Use of Laboratory Animals (23). Procedures of animal experiments were approved by The Ethics Committee of the Laboratory Animal Care and Welfare, Zhejiang Academy of Medical Sciences (Zhejiang, China).

Animals. A total of 48 male Sprague Dawley (SD) rats $(220 \pm 5 \mathrm{~g})$ in 8 -week old were purchased from the Laboratory Animal Center of Zhejiang Province (Hangzhou, China) and bred in house. All rats were housed in a specific pathogen free facility under a 12-h light/dark cycle in an ambient temperature of $22-26^{\circ} \mathrm{C}$ and relative humidity of $\sim 55 \%$. Animals were fed standard laboratory rat chow. Animals were provided food and water ad libitum.

Experimental protocols. SD rats were divided into three groups (each, $\mathrm{n}=6$ ) that received 0 (control), 1 and $10 \mathrm{mg} / \mathrm{kg}$ PFOS (Sigma Aldrich; Merck KGaA). The rats were intraperitoneally (I.P) injected at $1 \mathrm{~cm}$ to the left of the midline of the lower abdomen with PFOS every other day for 14 days. The dose, period and drug-delivery way of PFOS was selected based on previous reports $(24,25)$. On day 14 , all animals were placed on the operating table and immobilized with ketamine $(40 \mathrm{mg} / \mathrm{kg}$; intramuscular injection in the front of the thigh) and xylazine ( $5 \mathrm{ml} / \mathrm{kg}$; I.P) anesthesia, after which $5 \mathrm{ml}$ blood was obtained from the aorta abdominalis. After blood and heart tissues were obtained, rats were sacrificed by exsanguination from the carotid artery. Rat hearts and blood were used for biochemical and pathological assays (Fig. 1A). One sample from each group was used for preliminary experiments.
Body weight and hearts weight determinations. Changes of body weight in each group were measured every other day until the 14th day. At the end of the PFOS treatment, the rats were sacrificed and hearts were quickly moved, carefully blotted dry and weighed. The percentage of heart weight to body weight was calculated as follow: (heart weight/body weight) $\mathrm{x} 100 \%$.

Heart tissues and blood biochemical assays. Blood samples were obtained from the abdominal aorta and left to stand at room temperature for $1 \mathrm{~h}$, and then at $4^{\circ} \mathrm{C}$ for $2 \mathrm{~h}$, followed by $3,000 \times \mathrm{g}$ centrifugation for $10 \mathrm{~min}$ at $4^{\circ} \mathrm{C}$. The supernatant was used for serum lactic dehydrogenase (cat. no. 201902; LDH), creatine kinase (cat. no. 201823; CK) and creatine kinase-isoenzyme-MB (cat. no. 201811; CK-MB) measurements. The collected heart tissues were homogenized and centrifuged at $3,200 \times \mathrm{g}$ for $30 \mathrm{~min}$ at $4^{\circ} \mathrm{C}$. The supernatant was harvested for cardiac troponin-T (cat. no. 201904; cTn-T) measurement. All biomarkers were determined using commercial ELISA kits (BD Biosciences).

Histological analysis. The isolated hearts were fixed in $4 \%$ paraformaldehyde for $24 \mathrm{~h}$ at room temperature and embedded in paraffin for histological analysis. Next, samples were cut into $5-\mu \mathrm{m}$ sections and heated at $65^{\circ} \mathrm{C}$ for $20 \mathrm{~min}$. Slides were then deparaffinized with xylene and dehydrated in a grade series of ethanol through 70, 80, 90, 95 and 100\%. The slices were stained with Masson for $10 \mathrm{~min}$ at room temperature to evaluate fibrosis and stained with wheat germ agglutinin (WGA) for $15 \mathrm{~min}$ at $37^{\circ} \mathrm{C}$ to analyze myocardial hypertrophy. Images were captured (magnification, x200 or x400) under a light microscope (Leica Microsystems $\mathrm{GmbH}$ ). The fibrosis area of heart tissues and the cardiomyocyte cross-sectional area were measured using Image-Pro Plus software version 6.0 (Media Cybernetics, Inc.). The percentage of fibrotic areas were calculated.

Terminal deoxynucleotidyl transferase dUTP nick end labeling (TUNEL) staining. For all groups, $5-\mu \mathrm{m}$ sections were stained using the In Situ Cell Death kit-TMR (cat. no. MK1012; Wuhan Boster Biological Technology, Ltd.) overnight at $4^{\circ} \mathrm{C}$. The process of TUNEL staining were performed according to the manufacturer's instructions. The slides were incubated with TUNEL reaction mixture for $1 \mathrm{~h}$ at $37^{\circ} \mathrm{C}$ before being rinsed 3 times with PBS. The nucleus was stained by DAPI (1 $\mu \mathrm{g} / \mathrm{ml}$; cat. no. C1002; Beyotime Institute of Biotechnology) for $10 \mathrm{~min}$ at room temperature. A drop of antifade mounting medium (cat. no. P0098; Beyotime Institute of Biotechnology) was added for $5 \mathrm{~min}$ at room temperature. Images were taken with a fluorescence microscope and the number of TUNEL-positive cells in five random views were selected in each sample. Cells were counted by ImageJ analysis software (version 1.51; National Institutes of Health).

Immunohistochemical analysis. For immunohistochemical assay, heart tissues were fixed in $4 \%$ paraformaldehyde for $24 \mathrm{~h}$ at room temperature and embedded in paraffin for immunohistochemical analysis. Sections were blocked using 5\% goat serum (cat. no. G1209; Wuhan Boster Biological Technology, Ltd.) and incubated with specific primary antibodies overnight 

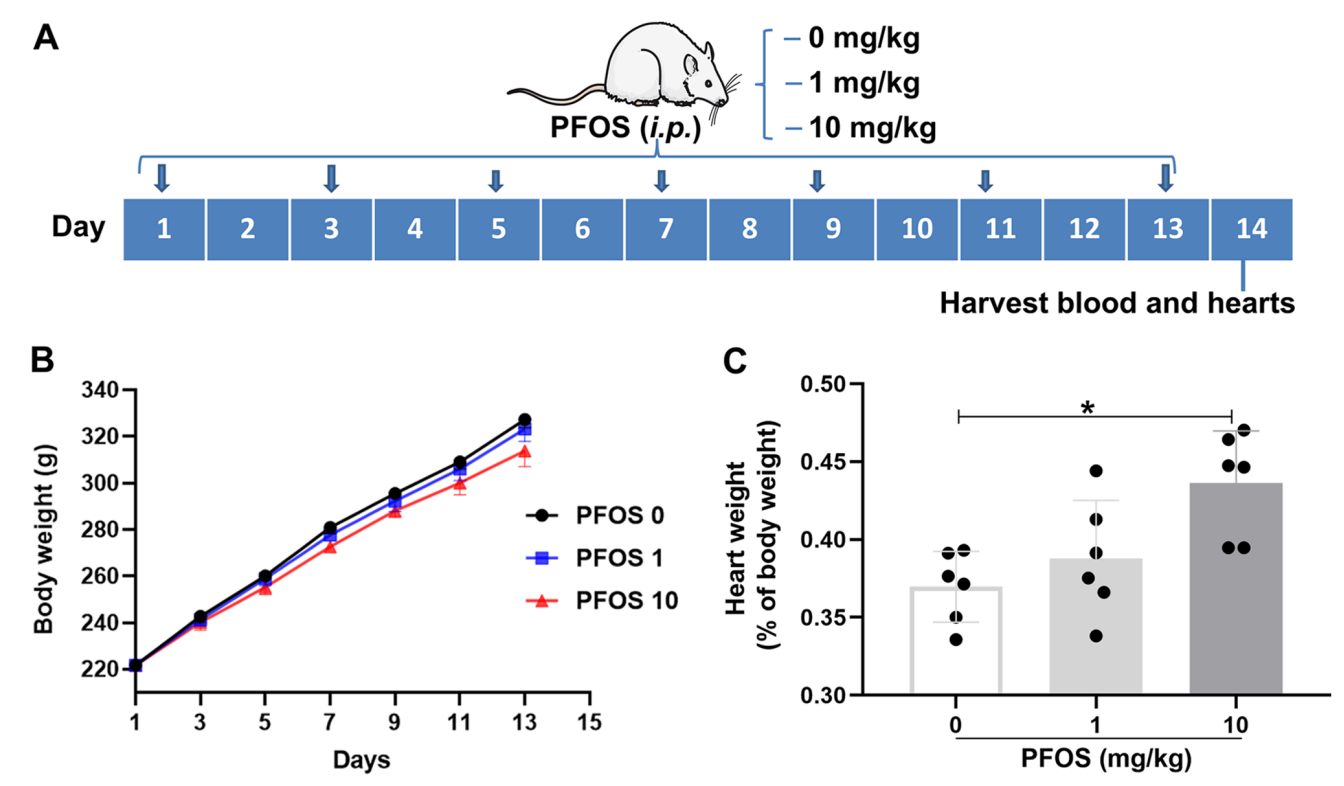

Figure 1. Illustration of experimental schedule and general indexes of rats. (A) Experimental schedule schematic. Sprague-Dawley rats received 0, 1 and $10 \mathrm{mg} / \mathrm{kg}$ perfluorooctane sulfonate (i.p.) every two days as indicated by the arrows. On day 14, blood and hearts were collected. (B) Body weights of the rats were recorded every other day for 2 weeks. (C) Relative heart weight (percentage ratio of heart weight to body weight). $n=6$. ${ }^{*} \mathrm{P}<0.05$ as indicated. PFOS, perfluorooctane sulfonate; i.p., intraperitoneal injection.

at $4^{\circ} \mathrm{C}$, such as IL-1 $\beta$ Rabbit $\mathrm{mAb}(1: 800$; cat. no. SRP8033; Sigma-Aldrich; Merck KGaA) and TNF- $\alpha$ Rabbit mAb (1:500; cat. no. ab6671, Abcam). The sections were washed with PBS three times, followed by staining with horseradish peroxidase-conjugated secondary anti-rabbit IgG (1:5,000; cat. no. GB23303; Wuhan Boster Biological Technology, Ltd.) for $2 \mathrm{~h}$ at room temperature and visualizing with substrate DAB. Images were obtained and captured (magnification, x200) using a fluorescent microscope (Leica Microsystems $\mathrm{GmbH}$ ). The number of positive cells was analyzed by ImageJ analysis software (version 1.51; National Institutes of Health).

Western blotting. Protein from myocardial tissue was extracted using the cell and tissue total protein extraction kit (cat. no. KC415; Shanghai Kang Cheng Bioengineering Co., Ltd.). Protein concentration was quantified using the bicinchoninic acid (BCA) protein assay kit (cat. no. P0010; Beyotime Institute of Biotechnology) and was diluted to the same concentration with 5X loading buffer (cat. no. P0015L; Beyotime Institute of Biotechnology). A total of $50 \mu \mathrm{g}$ protein was separated using $15 \%$ SDS-PAGE. Protein was transferred to polyvinylidene fluoride membrane and blocked in 5\% skimmed milk in Tris-buffered saline for $90 \mathrm{~min}$ at room temperature. After blocking, the membranes were incubated overnight with primary antibodies against p53 mouse mAb (1:1,000; cat. no. 2524; Cell Signaling Technology, Inc.), anti-Bax antibody (1:800; cat. no. A00183; Wuhan Sanying Biotechnology) and GAPDH mouse mAb (1:3,000; cat. no. G3214; Bioworld Technology, Inc.) overnight at $4^{\circ} \mathrm{C}$. Following washing with Tris-buffered saline containing $1 \%$ Tween, membranes were incubated for $2 \mathrm{~h}$ with horseradish peroxidase-conjugated IgG (1:5,000, cat. no. GB23303; Wuhan Boster Biological Technology, Ltd.) secondary antibodies at room temperature for $2 \mathrm{~h}$. The specific protein bands were visualized using an enhanced chemiluminescence detection kit (cat. no. 33021;
Boster Biological Technology, Ltd.). The intensity of each band was quantified using Quantity One software version 6 (Bio-Rad Laboratories, Inc.).

Statistical analyses. Data are presented as mean \pm standard deviation. Values from three groups were analyzed using one-way ANOVA followed by Student-Newman-Keuls multiple comparison post hoc test. Statistical analysis was performed in GraphPad Software (Prism Version 8.01; GraphPad Software, Inc.). P $<0.05$ was considered to indicate a statistically significant difference.

\section{Results}

Effect of PFOS on body weight and associated heart weight in rats. Body weight in 0 (control), 1 and $10 \mathrm{mg} / \mathrm{kg}$ PFOS groups were recorded every two days for 14 days. There was no significant difference in the body weight of rats among the three groups, though a slight reduction was observed between control and $10 \mathrm{mg} / \mathrm{kg}$ PFOS group (Fig. 1A). As presented in Fig. 1C, the percentages ratio of heart weight to body weight demonstrated no significant increase in $1 \mathrm{mg} / \mathrm{kg}$ group ( $P>0.05)$, but were significantly increased in the $10 \mathrm{mg} / \mathrm{kg}$ PFOS group compared with control group $(0.44 \pm 0.03 \%$ vs. $0.37 \pm 0.02 \%$; P $<0.05$; Fig. 1C).

PFOS-induced myocardial injury in rats. In order to detect the toxic effect of PFOS on the heart, cTn-T, LDH, CK and CK-MB, which are markers for myocardial injury, were measured in rats. In the $1 \mathrm{mg} / \mathrm{kg}$ PFOS group, only the level of cTn-T in heart tissues was significantly elevated compared with control group ( $\mathrm{P}<0.05 ;$ Fig. 2A). Whereas in the $10 \mathrm{mg} / \mathrm{kg}$ PFOS group, cTn-T, LDH, CK and CK-MB levels were all significantly increased when compared with the control group $(\mathrm{P}<0.05$; Fig. 2A-D). These results suggested that $10 \mathrm{mg} / \mathrm{kg}$ 

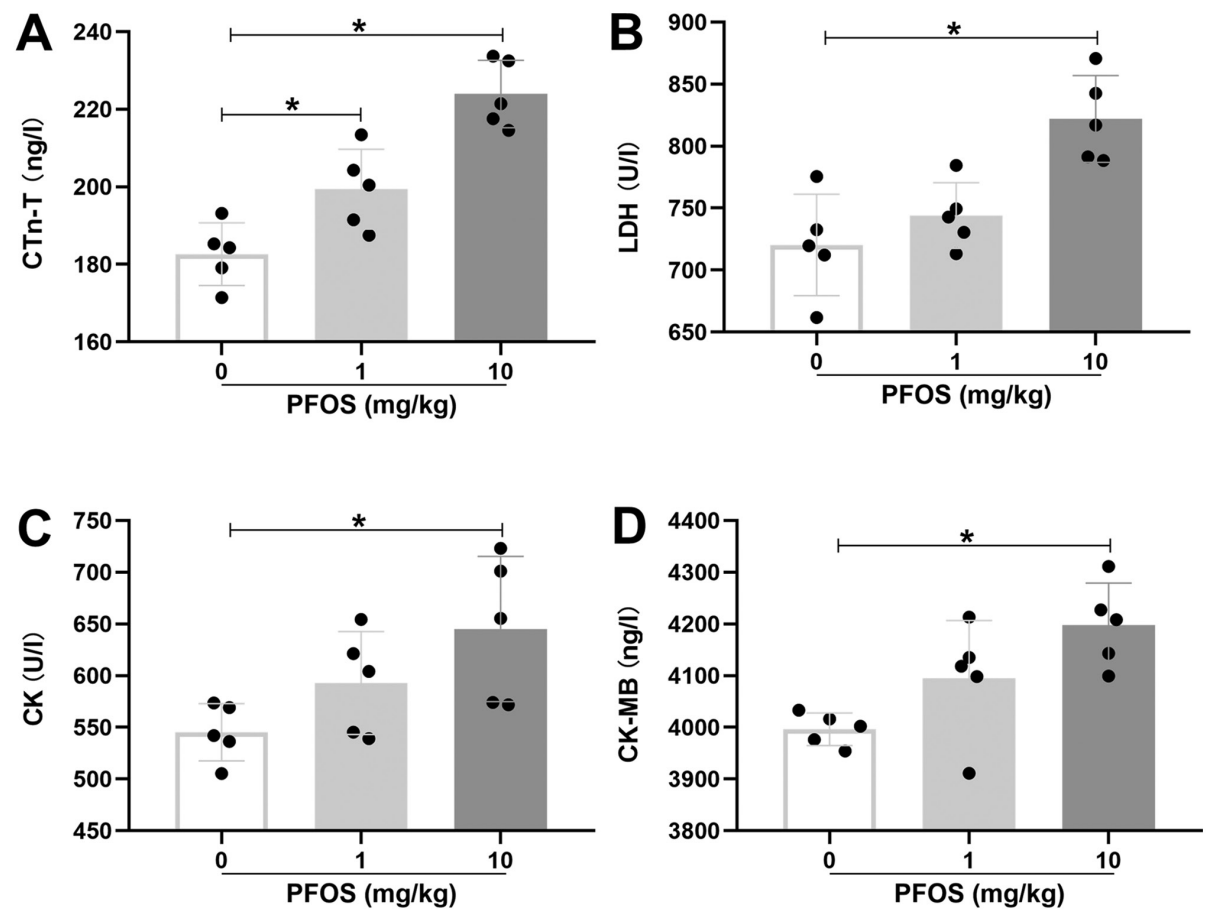

Figure 2. PFOS induces myocardial injury in rats. Markers of myocardial damage in cardiac tissues and serum were measured. (A) Expression levels of CTn-T in the cardiac tissues were determined. Contents of (B) LDH, (C) CK and (D) CK-MB in rat serum was measured. $\mathrm{n}=5$. ${ }^{*} \mathrm{P}<0.05$ as indicated. PFOS, perfluorooctane sulfonate; cTn-T, cardiac troponin-T; LDH, lactic dehydrogenase; CK, creatine kinase; MB, isoenzyme-MB.

PFOS could induce significant myocardial injury in rats. In addition, gavage administration was selected to test whether PFOS could elevate myocardial injury in rats. There were no significant differences between gavage administration and intraperitoneal injection administration on cTn-T, LDH, $\mathrm{CK}$ and $\mathrm{CK}-\mathrm{MB}$ expression levels among the three groups (P>0.05; Fig. S1).

To determine the toxicological effect of PFOS on rat hearts over a longer duration, PFOS was administrated every other day for 28 days to model sub-chronic exposure. The levels of cTn-T, $\mathrm{LDH}, \mathrm{CK}$ and CK-MB were examined after 28 days of PFOS exposure. The $10 \mathrm{mg} / \mathrm{kg}$ PFOS group demonstrated significantly elevated levels of cTn-T, LDH, CK and CK-MB compared with the $0 \mathrm{mg} / \mathrm{kg}$ PFOS group $(\mathrm{P}<0.05$; Fig. S2). The expression level of cTn-T was also significantly increased in the $1 \mathrm{mg} / \mathrm{kg}$ PFOS group compared with the $0 \mathrm{mg} / \mathrm{kg}$ PFOS group $(\mathrm{P}<0.05$; Fig. S2A). However, there were no significant differences in expression levels of $\mathrm{LDH}, \mathrm{CK}$ and CK-MB between the $1 \mathrm{mg} / \mathrm{kg}$ PFOS group and $0 \mathrm{mg} / \mathrm{kg}$ PFOS group (P>0 05 , Fig. S2B-D).

PFOS is associated with cardiac fibrosis and myocardiac hypertrophy in rats. To further determine the toxic influence of PFOS on heart tissues in rats, Masson and WGA staining were performed. The rats in the $1 \mathrm{mg} / \mathrm{kg}$ PFOS group exhibited no significant increase in cardiac fibrosis and hypertrophy when compared with the control group ( $\mathrm{P}>0.05$; Fig. 3A and $\mathrm{B}$ ). However, the fibrosis area and the myocyte cross-sectional area were markedly upregulated in rat hearts exposed to $10 \mathrm{mg} / \mathrm{kg}$ PFOS compared with the control group (fibrosis area, $7.2 \pm 1.2 \%$ vs. $0.0 \pm 0.0 \%$; $\mathrm{P}<0.05$; Fig. $3 \mathrm{~A}$ and $\mathrm{C}$; cross-sectional area, $412.6 \pm 47.4 \mu \mathrm{m}^{2}$ vs. $212.4 \pm 12.0 \mu \mathrm{m}^{2}$; $\mathrm{P}<0.05$; Fig. $3 \mathrm{~B}$ and D). These results were matched by the aforementioned changes in the percentage of heart weight to body weight.
PFOS is associated with myocardial apoptosis in rats. The cardiotoxicity effect of PFOS on apoptosis was explored using TUNEL staining. As presented in Fig. 4A and B, rats subjected to $1 \mathrm{mg} / \mathrm{kg}$ PFOS exhibited no difference in myocardial apoptosis compared with the control group $(\mathrm{P}>0.05)$. In addition, in the $10 \mathrm{mg} / \mathrm{kg}$ PFOS group, the percentage of TUNEL-positive nuclei were significantly increased compared with the control group $(\mathrm{P}<0.05)$. Furthermore, the expression levels of p53 and Bax were significantly upregulated in the $10 \mathrm{mg} / \mathrm{kg}$ PFOS group compared with the control group $(\mathrm{P}<0.05$; Fig. $4 \mathrm{C}$ and $\mathrm{D})$, and p53 protein was also significantly upregulated in the $1 \mathrm{mg} / \mathrm{kg}$ PFOS group ( $\mathrm{P}<0.05$; Fig. 4C and D).

PFOS is associated with inflammatory infiltration in rat heart tissues. The influence of PFOS on the cardiac inflammation in rats was investigated. Immunohistochemical staining for pro-inflammatory cytokine IL-1 $\beta$ indicated that the expression profile of IL-1 $\beta$ was significantly increased in the $10 \mathrm{mg} / \mathrm{kg}$ PFOS group compared with the control group (6.60 \pm 0.65 vs. $3.84 \pm 0.76 \mathrm{pg} / \mathrm{mg}$ protein; $\mathrm{P}<0.05$; Fig. $5 \mathrm{~A}$ and $\mathrm{B})$. Consistent with the apparent IL-1 $\beta$ accumulation, there was also a significant upregulation of TNF- $\alpha$ expression in the $10 \mathrm{mg} / \mathrm{kg}$ PFOS group compared with the control group (1.41 \pm 0.09 vs. $1.00 \pm 0.10 ; \mathrm{P}<0.05$; Fig. $5 \mathrm{~A}$ and $\mathrm{C}$ ). In addition, there was little expression of IL-1 $\beta$ and TNF- $\alpha$ in heart tissues exposed to $1 \mathrm{mg} / \mathrm{kg}$ PFOS $(\mathrm{P}>0.05)$.

\section{Discussion}

The current study revealed several main findings. Firstly, exposure of PFOS at the dosage of $10 \mathrm{mg} / \mathrm{kg}$ caused myocardial damage in rats. Secondly, $10 \mathrm{mg} / \mathrm{kg}$ PFOS significantly increased cardiac fibrosis and myocardiac hypertrophy in rats. 
A PFOS (mg/kg)

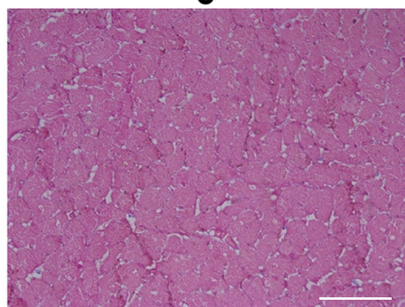
1 10

B
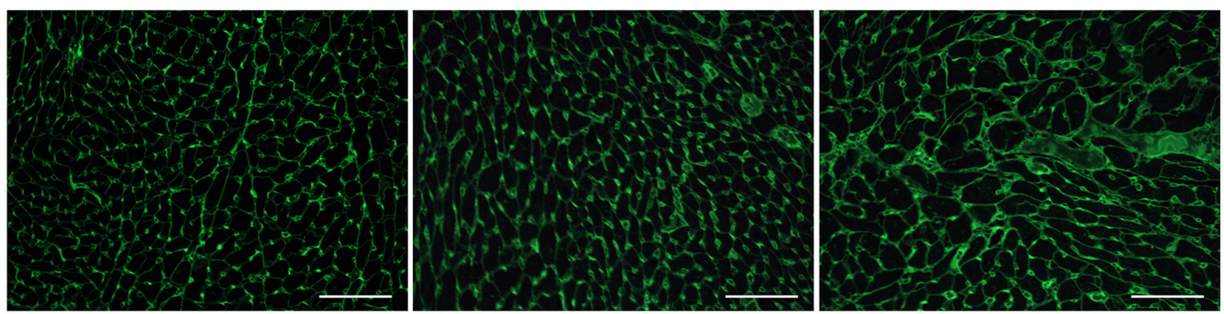

C
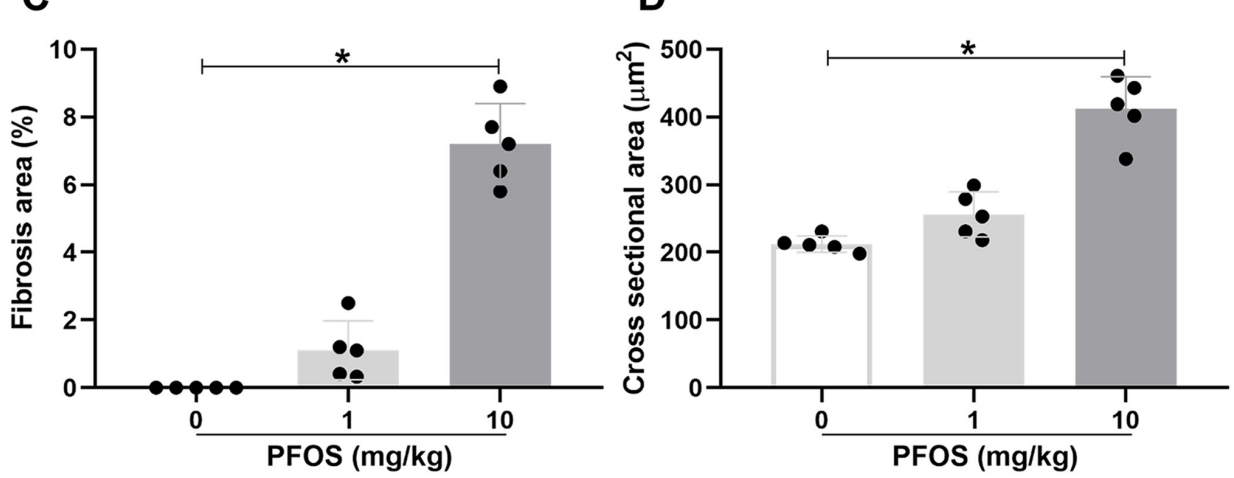

Figure 3. PFOS is associated with cardiac fibrosis and myocardiac hypertrophy in rats. (A) Masson staining and (B) wheat germ agglutinin staining of the rat hearts exposed to different doses of PFOS. Statistical graph of the (C) fibrosis area and the (D) cardiomyocyte cross-sectional area. White arrows point out Masson-positive regions (A magnification, x400; scale bars, $50 \mu \mathrm{m}$. B magnification, x200, scale bars, $100 \mu \mathrm{m}$ ). $\mathrm{n}=5$. * $<0.05$. PFOS, perfluorooctane sulfonate.

Finally, it was demonstrated that $10 \mathrm{mg} / \mathrm{kg}$ PFOS treatment upregulated myocardial apoptosis and expression levels of IL- $1 \beta$ and TNF- $\alpha$ in heart tissue of rats.

PFOS, a type of fluorine-saturated eight-carbon compound, is a persistent, bioaccumulative and organic pollutant as a result of its ubiquitous distribution and extreme stability (26). For humans, dietary intake is the main source of exposure to PFOS. Pollution data by the National Health and Nutrition Examination Survey has demonstrated that serum concentrations of PFOS range from 0.8-0.9 $\mu \mathrm{M}$, while an increased concentration (0.3-6.9 $\mu \mathrm{M}$ ) has been detected in Minnesota Mining and Manufacturing company employees $(3,27)$. Serum PFOS concentrations are generally far lower compared with $1 \mathrm{mg} / \mathrm{kg}$ in this study. However, based on the average weight of adults in China $(60 \mathrm{~kg})$ and their average blood content $(\sim 6,400 \mathrm{ml})$, the serum PFOS concentration of fluorination plant workers is $1,386 \mathrm{ng} / \mathrm{ml}$ (28); therefore, the average concentration of PFOS has been calculated to reach $0.1 \mathrm{mg} / \mathrm{kg}$ body weight. Studies have demonstrated that concentrations of PFOS that are lower than the oral PFOS $\mathrm{LD}_{50}$ of $250 \mu \mathrm{g} / \mathrm{g}$ (29) were associated with significant injury in rats or in mice $(4,25)$. In the current study, the dosage of $10 \mathrm{mg} / \mathrm{kg}$ was a little higher compared with the accumulated doses in human blood.
The cardiovascular toxicity of PFOS has been rudimentarily studied in vitro and in vivo. Harada et al (30) reported that the action potential duration and peak potential were markedly reduced when exposed to PFOS in guinea-pig ventricular myocytes (30). The sinus venosus-bulbus arteriosus distance was also demonstrated to be increased in a marine medaka when exposed to PFOS (9). Moreover, PFOS can enlarge the right atrium of mice and rats (31). PFOS not only affects heart malformation, but also heart function. PFOS exposure also changes heart rates in zebrafish embryos (32). Our previous study demonstrated that PFOS induced toxicity of ESCs through mitochondrial structure injury and abnormal $\mathrm{Ca}^{2+}$ shuttle (33). However, there is only a small amount of research performed to assess the cardiovascular toxicity of PFOS in rats. To investigate what effects PFOS exposure exerted on cardiac toxicity, the present study detected biochemical indices and pathological changes in rats. It was demonstrated that PFOS treatment augmented the percentage of heart to body weight in rats. Moreover, $10 \mathrm{mg} / \mathrm{kg}$ PFOS induced significant cardiac fibrosis and myocardiac hypertrophy that was matched by increased biochemical indices associated with myocardial damage in rats. The results of the present study suggested that PFOS at a dosage of $10 \mathrm{mg} / \mathrm{kg}$ could exert a pronounced cardiotoxicity in rats; however the mechanism underlying PFOS toxicity in the cardiovascular system remains unclear. 


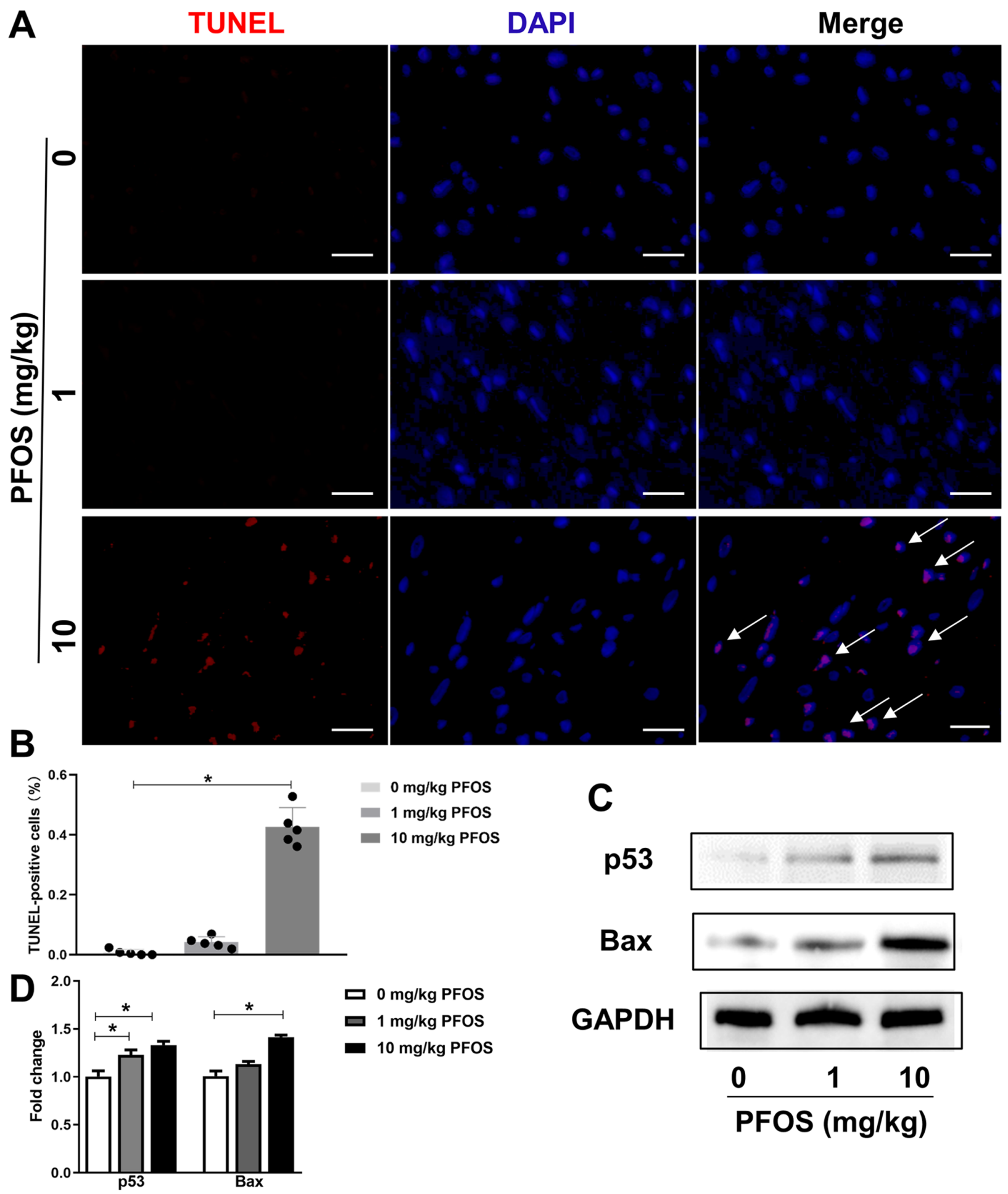

Figure 4. PFOS is associated with myocardial apoptosis in rats. (A) Representative images stained with TUNEL (red) and Dapi (blue). White arrows indicate TUNEL-positive cells (scale bar, $50 \mu \mathrm{m}$; magnification, $\mathrm{x} 400$ ). (B) Quantitative analysis of the percentage of TUNEL-positive cells in rat hearts (n=5). (C) Western blotting of $\mathrm{p} 53$ and Bax in rat heart. (D) Quantitative analysis of the effect of PFOS treatment effects on p53 and Bax ( $\mathrm{n}=3$ ). ${ }^{*} \mathrm{P}<0.05$ as indicated. PFOS, perfluorooctane sulfonate.

Apoptosis is an important process in various human diseases and has been implicated in PFOS toxicity (34). A small number of basal studies have been carried out to examine the toxicity of PFOS associated with apoptosis (35-37). PFOS treatment can induce cell apoptosis in murine N9 cells (35) and in hepatoma Hep G2 cells (36). It can also upregulate the number of apoptotic cells in liver of adult rats (37). However, PFOS exposure has been reported to influence the protein and mRNA expression levels of Bax and Bcl-2 in adults (38). In addition, the genes associated with apoptosis, such as p53 and Bax, were significantly upregulated in zebrafish embryos that were raised in an environment with PFOS, the mechanism of which was associated with ROS generation and MAPK activity (21). A previous 


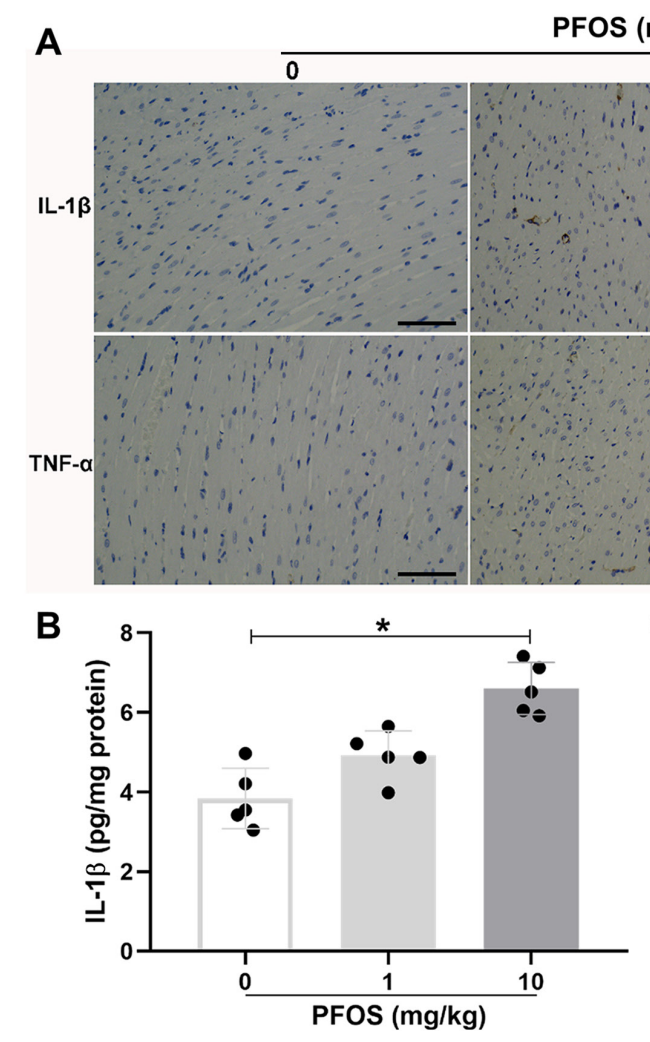

PFOS $(\mathrm{mg} / \mathrm{kg})$
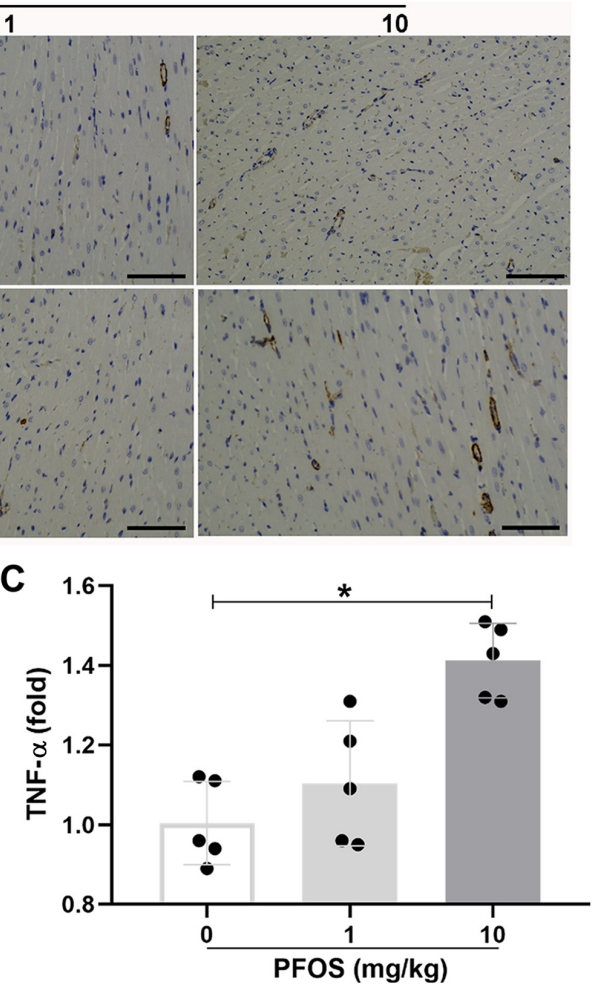

Figure 5. PFOS is associated with inflammatory infiltration in rat heart tissues. (A) Immunohistochemistry staining demonstrated the effect of different doses of PFOS on protein levels of IL-1 $\beta$ and TNF- $\alpha$ (magnification, x200; scale bars, $100 \mu \mathrm{m}$ ). Protein expression levels of (B) IL-1 $1 \beta$ and (C) TNF- $\alpha$ were quantitatively analyzed. $\mathrm{n}=5$. ${ }^{*} \mathrm{P}<0.05$ as indicated. $\mathrm{PFOS}$, perfluorooctane sulfonate.

study revealed that PFOS exposure results in the apoptosis of rat cardiocytes via TUNEL analysis (18). In line with this study, the current study revealed that $10 \mathrm{mg} / \mathrm{kg}$ PFOS induced increases of TUNEL-positive cells, p53 and Bax protein expression, suggesting an increase in apoptosis in the heart tissues of adult rats.

Increasing evidence has demonstrated that inflammation serves a notable role in Polyfluoroalkyl chemical-induced toxicity $(22,39)$. Perfluorononanoic acid is hypothesized to increase liver weight and upregulate large quantities of IL-1 $\beta$ and TNF- $\alpha$ (39). In addition, it is reported that PFOS exposure leads to hepatocyte proliferation accompanied by an increase of serum IL-6 and TNF- $\alpha$ (22). On the contrary, clinical research has revealed that lower gut inflammation is closely associated with higher perfluoroalkyl substances exposure, which can be regarded as a risk factor for inflammatory bowel disease (40). However, whether pro-inflammatory cytokines are involved in PFOS-stimulated cardiac toxicity remains unclear. Notably, the results of the current study revealed that proinflammatory cytokines IL- $1 \beta$ and TNF- $\alpha$ were significantly elevated in rat heart tissues after exposure to $10 \mathrm{mg} / \mathrm{kg}$ PFOS.

Previous studies have reported that the exposure of female rats to perfluorooctane sulfonate increased the estrogen receptor $\alpha(E R \alpha)$ expression, suggesting that PFOS acts as estrogenic compounds to activate $\mathrm{ER} \alpha(41,42)$. In adult male and female B6C3F1 mice, daily PFOS exposure could induce immunotoxicity, with certain differences between male mice and female mice being identified in regards to immune parameters (43). Further study should be conducted to provide evidence towards the difference of male and female rats in cardiac toxicity. In addition, as an organic compound, the concentration of PFOS in heart tissue needs to be detected with high performance liquid chromatography in future experiments.

In summary, the current study demonstrated that PFOS exposure caused pathological changes, reflected by cardiac fibrosis and myocardial hypertrophy in the hearts of adult rats, which was possibly associated with an increase in apoptosis and proinflammatory cytokines, such as IL-1 $\beta$ and TNF- $\alpha$. The present study provided preliminary data for further study of cardiovascular system subjected to a PFOS challenge.

\section{Acknowledgements}

Not applicable.

\section{Funding}

This study was supported by The Medical Health Science and Technology Project of Zhejiang provincial Health Commission (grant no. 2018KY148) and The Medical Health Science and Technology Program of Hangzhou (grant no. 20190551).

\section{Availability of data and materials}

The datasets used and/or analyzed during the current study are available from the corresponding author on reasonable request. 


\section{Authors' contributions}

JY and MG designed the current study and wrote the manuscript. DX, LL and LT performed experiments and analyzed data. DX and JY confirmed the authenticity of all the raw data. All authors have read and approved the final manuscript.

\section{Ethics approval and consent to participate}

Procedures of animal experiments were approved by The Ethics Committee of Laboratory Animal Care and Welfare, Zhejiang Academy of Medical Sciences (Zhejiang, China).

\section{Patient consent for publication}

Not applicable.

\section{Competing interests}

The authors declare that they have no competing interests.

\section{References}

1. Wang G, Sun S, Wu X, Yang S, Wu Y, Zhao J, Zhang H and Chen W: Intestinal environmental disorders associate with the tissue damages induced by perfluorooctane sulfonate exposure. Ecotoxicol Environ Saf 197: 110590, 2020.

2. Gao Y, Guo X, Wang S, Chen F, Ren X, Xiao H and Wang L: Perfluorooctane sulfonate enhances mRNA expression of PPAR $\gamma$ and ap2 in human mesenchymal stem cells monitored by long-retained intracellular nanosensor. Environ Pollut 263: 114571, 2020.

3. Olsen GW, Burris JM, Ehresman DJ, Froehlich JW, Seacat AM, Butenhoff JL and Zobel LR: Half-life of serum elimination of perfluorooctanesulfonate, perfluorohexanesulfonate, and perfluorooctanoate in retired fluorochemical production workers. Environ Health Perspect 115: 1298-1305, 2007.

4. Lau C, Thibodeaux JR, Hanson RG, Rogers JM, Grey BE, Stanton ME, Butenhoff JL and Stevenson LA: Exposure to perfluorooctane sulfonate during pregnancy in rat and mouse. II: Postnatal evaluation. Toxicol Sci 74: 382-392, 2003.

5. UNEP: The stockholm convention-national implementation plans-addressing COP 4 amendments. http://chm.pops. int/Implementation/NIPs/NIPTransmission/tabid/253/Default. aspx. (accessed on September 7th 2019), 2016.

6. Xie S, Wang T, Liu S, Jones KC, Sweetman AJ and Lu Y: Industrial source identification and emission estimation of perfluorooctane sulfonate in China. Environ Int 52: 1-8, 2013.

7. Wang S, Huang J, Yang Y, Hui Y, Ge Y, Larssen T, Yu G, Deng S, Wang B and Harman C: First report of a Chinese PFOS alternative overlooked for 30 years: Its toxicity, persistence, and presence in the environment. Environ Sci Technol 47: 10163-10170, 2013.

8. Jin H, Lin S, Dai W, Feng L, Li T, Lou J and Zhang Q: Exposure sources of perfluoroalkyl acids and influence of age and gender on concentrations of chlorinated polyfluorinated ether sulfonates in human serum from China. Environ Int 138: 105651, 2020.

9. Huang Q, Fang C, Wu X, Fan J and Dong S: Perfluorooctane sulfonate impairs the cardiac development of a marine medaka (Oryzias melastigma). Aquat Toxicol 105: 71-77, 2011.

10. Chan E, Burstyn I, Cherry N, Bamforth F and Martin JW: Perfluorinated acids and hypothyroxinemia in pregnant women. Environ Res 111: 559-564, 2011.

11. Elcombe CR, Elcombe BM, Foster JR, Chang SC, Ehresman DJ and Butenhoff JL: Hepatocellular hypertrophy and cell proliferation in sprague-dawley rats from dietary exposure to potassium perfluorooctanesulfonate results from increased expression of xenosensor nuclear receptors PPAR $\alpha$ and CAR/PXR. Toxicology 293: 16-29, 2012.

12. Yang L, He L, Xue J, Ma Y, Xie Z, Wu L, Huang M and Zhang Z: Persulfate-based degradation of perfluorooctanoic acid (PFOA) and perfluorooctane sulfonate (PFOS) in aqueous solution: Review on influences, mechanisms and prospective. J Hazard Mater 393: 122405, 2020
13. Zhang YY, Tang LL, Zheng B, Ge RS and Zhu DY: Protein profiles of cardiomyocyte differentiation in murine embryonic stem cells exposed to perfluorooctane sulfonate. J Appl Toxicol 36: 726-740, 2016.

14. Cheng W, Yu Z, Feng L and Wang Y: Perfluorooctane sulfonate (PFOS) induced embryotoxicity and disruption of cardiogenesis. Toxicol In Vitro 27: 1503-1512, 2013.

15. Xia W, Wan Y, Li YY, Zeng H, Lv Z, Li G, Wei Z and Xu SQ: PFOS prenatal exposure induce mitochondrial injury and gene expression change in hearts of weaned SD rats. Toxicology 282: 23-29, 2011

16. Dong Y, Chen H, Gao J, Liu Y, Li J and Wang J: Molecular machinery and interplay of apoptosis and autophagy in coronary heart disease. J Mol Cell Cardiol 136: 27-41, 2019.

17. Tomei LD and Umansky SR: Apoptosis and the heart: A brief review. Ann N Y Acad Sci 946: 160-168, 2001.

18. Zeng HC, He QZ, Li YY, Wu CQ, Wu YM and Xu SQ: Prenatal exposure to PFOS caused mitochondia-mediated apoptosis in heart of weaned rat. Environ Toxicol 30: 1082-1090, 2015.

19. Zhang Y, Beesoon S, Zhu L and Martin JW: Isomers of perfluorooctanesulfonate and perfluorooctanoate and total perfluoroalkyl acids in human serum from two cities in North China. Environ Int 53: 9-17, 2013.

20. Shi X, Du Y, Lam PK, Wu RS and Zhou B: Developmental toxicity and alteration of gene expression in zebrafish embryos exposed to PFOS. Toxicol Appl Pharmacol 230: 23-32, 2008.

21. Shi $X$ and Zhou B: The role of Nrf2 and MAPK pathways in PFOS-induced oxidative stress in zebrafish embryos. Toxicol Sci 15: 391-400, 2010

22. Han R, Zhang F, Wan C, Liu L, Zhong Q and Ding W: Effect of perfluorooctane sulphonate-induced Kupffer cell activation on hepatocyte proliferation through the NF- $\kappa$ B/TNF- $\alpha /$ IL-6-dependent pathway. Chemosphere 200: 283-294, 2018

23. National Research Council: Guide for the Care and Use of Laboratory Animals: Eighth Edition. The National Academies Press, Washington, DC, 2011.

24. Chou HC, Wen LL, Chang CC, Lin CY, Jin L and Juan SH: From the cover: 1-carnitine via PPAR $\gamma$ - and sirt1-dependent mechanisms attenuates epithelial-mesenchymal transition and renal fibrosis caused by perfluorooctanesulfonate. Toxicol Sci 160: 217-229, 2017.

25. Wen LL, Lin CY, Chou HC, Chang CC, Lo HY and Juan SH: Perfluorooctanesulfonate mediates renal tubular cell apoptosis through PPARgamma inactivation. PLoS One 11: e0155190, 2016.

26. Zhang L, Duan X, Sun W and Sun H: Perfluorooctane sulfonate acute exposure stimulates insulin secretion via GPR40 pathway. Sci Total Environ 726: 138498, 2020.

27. Calafat AM, Wong LY, Kuklenyik Z, Reidy JA and Needham LL: Polyfluoroalkyl chemicals in the U.S. population: Data from the national health and nutrition examination survey (NHANES) 2003-2004 and comparisons with NHANES 1999-2000. Environ Health Perspect 115: 1596-1602, 2007.

28. Yan G: Environmental behavior and human exposure to perfluoroalkyl acids around a manufacturing facility in China. Graduate University of the Chinese Academy of Sciences, 2017.

29. 3M: Perfluorooctane Sulfonate: Current Summary of Human Sera, Health and Toxicology Data. 1stedition. U.S. Environmental Protection Agency, St. Paul, MN, pp1-129, 1999.

30. Harada K, Xu F, Ono K, Iijima T and Koizumi A: Effects of PFOS and PFOA on L-type Ca2+ currents in guinea-pig ventricular myocytes. Biochem Biophys Res Commun 329: 487-494, 2005.

31. Thibodeaux JR, Hanson RG, Rogers JM, Grey BE, Barbee BD, Richards JH, Butenhoff JL, Stevenson LA and Lau C: Exposure to perfluorooctane sulfonate during pregnancy in rat and mouse. I: Maternal and prenatal evaluations. Toxicol Sci 74: 369-381, 2003.

32. Huang H, Huang C, Wang L, Ye X, Bai C, Simonich MT, Tanguay RL and Dong Q: Toxicity, uptake kinetics and behavior assessment in zebrafish embryos following exposure to perfluorooctanesulphonicacid (PFOS). Aquat Toxicol 98: 139-147, 2010.

33. Tang LL, Wang JD, Xu TT, Zhao Z, Zheng JJ, Ge RS and Zhu DY: Mitochondrial toxicity of perfluorooctane sulfonate in mouse embryonic stem cell-derived cardiomyocytes. Toxicology 382: 108-116, 2017.

34. Xu M, Liu G, Li M, Huo M, Zong W and Liu R: Probing the cell apoptosis pathway induced by perfluorooctanoic acid and perfluorooctane sulfonate at the subcellular and molecular levels. J Agric Food Chem 68: 633-641, 2020. 
35. Zhang L, Li YY, Zeng HC, Li M, Wan YJ, Schluesener HJ, Zhang ZY and Xu SQ: Perfluorooctane sulfonate induces apoptosis in N9 microglial cell line. Int J Toxicol 30: 207-215, 2011.

36. Hu XZ and Hu DC: Effects of perfluorooctanoate and perfluorooctane sulfonate exposure on hepatoma Hep G2 cells. Arch Toxicol 83: 851-861, 2009.

37. Kim HS, Jun Kwack S, Sik Han E, Seok Kang T, Hee Kim S and Young Han S: Induction of apoptosis and CYP4A1 expression in sprague-dawley rats exposed to low doses of perfluorooctane sulfonate. J Toxicol Sci 36: 201-210, 2011.

38. Shen Y and White E: p53-dependent apoptosis pathways. Adv Cancer Res 82: 55-84, 2001.

39. Fang X, Zou S, Zhao Y, Cui R, Zhang W, Hu J and Dai J: Kupffer cells suppress perfluorononanoic acid-induced hepatic peroxisome proliferator-activated receptor $\alpha$ expression by releasing cytokines. Arch Toxicol 86: 1515-1525, 2012.

40. Xu Y, Li Y, Scott K, Lindh CH, Jakobsson K, Fletcher T, Ohlsson B and Andersson EM: Inflammatory bowel disease and biomarkers of gut inflammation and permeability in a community with high exposure to perfluoroalkyl substances through drinking water. Environ Res 181: 108923, 2020.
41. Qiu Z, Qu K, Luan F, Liu Y, Zhu Y, Yuan Y, Li H, Zhang H, Hai Y and Zhao C: Binding specificities of estrogen receptor with perfluorinated compounds: A cross species comparison. Environ Int 134: 105284, 2020.

42. Xu C, Jiang ZY, Liu Q, Liu H and Gu AH: Estrogen receptor beta mediates hepatotoxicity induced by perfluorooctane sulfonate in mouse. Environ Sci Pollut Res Int 24: 13414-13423, 2017.

43. Peden-Adams MM, Keller JM, Eudaly JG, Berger J, Gilkeson GS and Keil DE: Suppression of humoral immunity in mice following exposure to perfluorooctane sulfonate. Toxicol Sci 104: 144-154, 2008.

This work is licensed under a Creative Commons Attribution-NonCommercial-NoDerivatives 4.0 International (CC BY-NC-ND 4.0) License. 